Revue d'histoire de l'Amérique française

ZWB REVUE D.HISTOIRE DE L'AMÉRIQUE FRANÇAISE

\title{
Pour lire les Mémoires de Lionel Groulx (1878-1967)
}

\section{Benoît Lacroix}

Volume 24, numéro 3, décembre 1970

URI : https://id.erudit.org/iderudit/302993ar

DOI : https://doi.org/10.7202/302993ar

Aller au sommaire du numéro

Éditeur(s)

Institut d'histoire de l'Amérique française

ISSN

0035-2357 (imprimé)

1492-1383 (numérique)

Découvrir la revue

Citer cette note

Lacroix, B. (1970). Pour lire les Mémoires de Lionel Groulx (1878-1967). Revue

d'histoire de l'Amérique française, 24(3), 413-419.

https://doi.org/10.7202/302993ar d'utilisation que vous pouvez consulter en ligne.

https://apropos.erudit.org/fr/usagers/politique-dutilisation/ 


\title{
NOTE CRITIQUE \\ POUR LIRE LES MÉMOIRES DE LIONEL GROULX $(1878-1967) *$
}

\author{
Benô̂t LACROIX \\ Université de Montréal
}

L'édition intégrale des Mémoires du chanoine Lionel Groulx que publie la maison Fides de Montréal renvoie à un manuscrit de plus de 2675 pages rédigées à la main, à Montréal, parfois à Vaudreuil, de 1954 à 1967. C'est énorme, d'autant que l'auteur écrit entre-temps ses sept derniers livres et qu'il ne cesse de rééditer d'autres ouvrages souvent "revus et corrigés".

Il est né en 1878, il meurt en 1967, il raconte l'histoire de sa vie : en tout plus d'un siècle. Et quand il s'arrête, quelques semaines avant sa mort à Vaudreuil le 23 mai au matin, il voudrait bien continuer, mais... « J'entreprends ma $89^{\mathrm{e}}$ année. La fin approche. Je le sens en mon hésitation à prendre certaines attitudes, à me prononcer sur maints problèmes brûlants. J'ai trop conscience de n'être plus de ce temps, de risquer de me tromper.»

Ecrit à temps perdu, le manuscrit des Mémoires s'étale sur des pages d'occasion, versos des épreuves de ses livres, feuilles volantes dont le format varie souvent ; il se reprend, il se corrige, insère ici et là un nouveau paragraphe, réajuste ses phrases, supprime rarement. Toujours la même écriture anguleuse et volontaire, ponctuée avec force, qui révèle un homme persévérant, laborieux et surtout orienté.

Il adore écrire. Quand il commence ses mémoires, le 8 août 1954 , il a 76 ans accomplis. C'est dire qu'il peut compter sur l'acquis, d'autant plus que sa mémoire reste étonnante. Il n'a pas à inventer non plus : sa vie est remplie au maximum. Qu'il doute d'une date, d'un nom, aussitôt il peut recourir à ses livres, à d'autres sources, inédites, un journal de collège qui remonte à 1895 , un journal de voyage en 1906, un autre carnet de route en 1956, des cahiers de lecture, des plans, des brouillons, des essais

* NOTE BIO-BIBLIOGRAPHIQUE. - L'œuvre du chanoine Lionel Groulx. Témoignages. Bio-bibliographie. Publications de l'Académie canadienne-française (Montréal, 1964); Lionel Groulx, Classiques canadiens, 30 (Montréal, Fides, 1967) ; Lionel Groulx, ptre. L'homme - L'œuvre, L'Action Nationale, 57 (juin 1968): 830-1115; Guy Frégault, "Lionel Groulx" dans Revue d'histoire de l'Amérique française 22 (1968) : 3-16.

[ 413 ]

RHAF, vol. 24, no 3 (décembre 1970) 
jamais publiés, une vaste correspondance, une vingtaine de spicilèges fidèlement codifiés par des secrétaires consciencieuses et convaincues.

Il aime écrire comme il aime causer. Aussi, ceux qui ne l'ont pas connu et surtout ceux plus nombreux encore qui ne l'ont connu que par ouï-dire, s'étonneront qu'en 1958, alors qu'il arrive aux souvenirs plus faciles des années 1950, il ait hésité, qu'il ait eu peur même. Il a eu comme le pressentiment d'une nouvelle page d'histoire qu'il devrait laisser inachevée. Pourtant le cinquième tome des Mémoires est terminé; il a prévu selon son habitude d'écrire toujours à partir d'un plan rédigé à l'avance, qu'il en écrirait sept, au moins sept. Le demi-siècle (1950) lui paraît, historien qu'il est, comme un excellent point d'arrêt ; il vieillit, il a déjà tellement écrit, l'avenir lui appartient de moins en moins. Pour une fois, l'unique peut-être, il faut presque le pousser dans le dos. Des proches, des amis, de vrais amis, peu nombreux mais efficaces, le supplient. Lionel Groulx se remet à l'nuvre. Tant mieux et tant mieux ! Car les demiers tomes de ses Mémoires contiennent des pages qui rejoindront la majorité des lecteurs d'aujourd'hui. Ces mêmes lecteurs apprendront en plus pourquoi Lionel Groulx n'a pas voulu publier ses Mémoires de son vivant : simple discrétion, besoin d'être libre, des inimitiés inutiles, le combat qui est loin d'être terminé, la peur de compromettre l'essentiel de son message devant des interprétations dont il deviendrait finalement le seul responsable, avec l'obligation de refuser qu'on déforme sa pensée qui se fait de plus en plus inquiète.

Et si les Mémoires doivent être publiés un jour ?

J'y mets ces deux conditions:

Premièrement - Elle (: sa nièce et secrétaire depuis 1937, madame Juliette Rémillard) ne publiera chaque volume qu'après avoir pris l'avis - surtout pour les derniers - de personnes prudentes, averties (les membres de la Fondation Lionel-Groulx peutêtre) sur l'opportunité de la publication.

Deuxièmement - Mes Mémoires devront être publiés sans modifications et sans suppressions sans coups de ciseaux. Que ma nièce publie ellemême, ou vende mes Mémoires à quelque éditeur, je la prie de veiller scrupuleusement à cette expresse volonté de l'auteur. Ecrit à Vaudreuil, ce 18 août 1958. 
Ecrire des Mémoires quand on a déjà publié trente livres, est-ce abuser ? a-t-il du temps à perdre ? une réputation à arrondir? des idées à se faire pardonner. A son âge tient-on encore à ses opinions ?

Ecrire ses Mémoires ! J'y ai toujours vu la besogne d'un paresseux et d'un impuissant. Pourtant, j'écris les miens. La Providence a voulu qu'en ces soixanteseize ans, j'aie connu une période de fatigue, le médecin m'a condamné au repos forcé. Pour me divertir, j'écris mes Mémoires...

Il insiste, comme s'il avait besoin de se rassurer.

J'écris au fil de la plume, ne méprisant ni l'ordre, ni la logique, mais flirtant volontairement avec le facile, le laisser-aller où tente pourtant de se définir l'histoire que j'ai vécue : histoire des pensées, des mouvements, des espérances, des déceptions où la vie m'a jeté.

Littérature de divertissement, flirt d'écrivain ? Ne le prenons pas trop au mot. Les divertissements du géant ne seront jamais des jeux d'enfant. Lionel Groulx a été investi d'une "certaine mission"; on lui a dit, il le croit, et il veut tout simplement poursuivre, c'est-à-dire éveiller les jeunes et rassurer les autres, son "petit peuple" qui hésite:

...Si j'écris ces pages où je résume le travail, les soucis de ma vie, les idées que j'ai essayé de mettre en circulation, c'est avec l'espoir de continuer quelque peu, quand je n'y serai plus, ce pourquoi j'ai vécu. J'essaie de continuer ma mission, si tant est que cette mission fût celle que l'on a présumée.

L'historien Groulx ne peut pas oublier les autres, ses collègues qui pensent sur les documents:

Il sera peut-être bon, pour ceux qui, un jour ou l'autre, s'égareront en ces pages, de savoir que ce furent les courants de pensée d'une époque et quels débats, par exemple, pouvait soulever en ce tempslà, une œuvre de littérature régionaliste.

Que peut-il bien raconter pour en écrire aussi long ? C'est qu'il a été mêlé à tout ce que nous avons fait et dit et pensé depuis cent ans. Etudiant, prêtre, historien, idéologue, homme d'action, écrivain, orateur et à ses heures politicien, il aura tout vécu avec ardeur et audace, dans la pleine conscience d'être 
français et "curé" en Amérique anglophone. Son enfance, son passé familial, sa vie de collège, l'enseignement à l'université, ses voyages, ses rencontres, ses livres, ses amitiés, ses démêlés avec les chefs religieux et politiques de l'époque, tout y passe et l'on est entraîné d'un récit à l'autre avec le même plaisir d'apprendre ce qui va suivre. A côté des grandes fresques sur l'essor du nationalisme français en Amérique, sur les mœurs politiques et religieuses de son milieu immédiat, il y a la "célèbre" petite histoire locale, l'anecdote savoureuse, le récit intime, les secrets d'arrière-scène, le portrait des amis et de ceux qu'on aime moins, les rebuffades, les succès, les misères de la vieillesse, le document caché au fond du tiroir, et caetera.

Disons que ses amis sont assez bien protégés : il a pour eux des mots fraternels et parfois très émouvants. Comme il peut aimer, admirer, pardonner, s'étonner ! Mais les autres, les politiciens arrivistes, les “imbéciles" de l' "imbécillité de l'esprit de "parti", les "peureux" et tous les rêveurs en couleur, les "ceux qui" se sont moqués de lui, certains anciens maîtres-répétitcurs, quelques irresponsables bien identifiés, les universitäires de couloirs, certains évêques même, je vous assure qu'ils en attrapent au détour d'un récit qui promettait pourtant d'être inoffensif ! Sur le Pont d'Avignon tout le monde y passe. Quel écrivain redoutable il est! Griffe ici, griffe là, sous le velours d'une phrase ondulée, des espiègleries verbales qui frisent l'exagération. Bien sûr, il veut être charitable, il ne dévore jamais sa victime au complet, mais ce qui en reste !. . Quel contestataire de qualité il aura été dès les années 1920 ! Sans poings, sans dynamite, sans barbe, il avance, creuse sa route : il est seul, il crie seul, il n'a peur de personne, il ne craint ni pape, ni évêque, ni Anglais, ni Irlandais. Il dira un jour pompeusement et à sa manière que le ton des Mémoires trahit d'ailleurs souvent, qu'il veut être de la génération des vivants bien que trop souvent condamné malgré lui à vivre avec des morts. En vain il aura attendu, dit-il, le chef qui mette le fait français à sa place en Amérique du Nord. En vain ? Parce que chez nous, paraît-il, les meilleures causes sont trop vite politisées (allusion au Bloc Populaire) et perdent peu à peu leur caractère sacré.

Devrions-nous prévenir le lecteur ? Inutile. De toute manière il va nous heurter et lasser ceux qui ont la respiration courte. Doit-on plutôt invoquer la psychanalyse et conclure que le petit Lionel, orphelin de père à peine un mois après sa propre naissance, - un 13 ! n'a jamais pu oublier ou plutôt n'a jamais voulu oublier que son père ne savait ni lire ni écrire, "pauvre enfant donné à l'âge de cinq ans à une famille d'étrangers" ? Est-ce 
si anormal qu'à 76 ans il se souvienne avec mélancolie, mais non sans une certaine fierté, des jours où pour se faire un peu de sous il allait vendre des fleurs à Dorion ? Comme tant d'autres de son espèce, l'enfance l'aura marqué à jamais. Il écrirait ses Mémoires pour exorciser son passé ? On peut tout supposer, pourvu qu'on dise qu'il veut avant tout épargner aux siens les mêmes humiliations.

Les grands moments de ses souvenirs ? Au-delà de l'enfance à la fois pittoresque et pénible, la vie au Collège, le récit de sa vocation sacerdotale, les aventures de sa carrière d'historien, son engagement social, la naissance du Devoir, l'histoire du mouvement nationaliste, ses livres, ses démêlés avec les "grands" de l'époque, ses défis, des amitiés perdues, des visiteurs inattendus, et combien d'autres faits encore que les Mémoires nous rappellent, toujours « à sa manière ». Il ne connaît pas les raccourcis de la syntaxique moderne, mais il écrit une langue qui toujours respire la perfection et la noblesse.

La grande qualité de ces Mémoires peu objectifs comme il se doit, est dans la franchise de leur ton autant que dans la beauté de leur style. On pourra discuter des options et des jugements de Lionel Groulx, critiquer ses attitudes, on pourra même penser qu'il a été conditionné par ses amitiés plus qu'il ne le faut, on enterrera sûrement plusieurs de ses pages d'histoire, - il en a toujours été ainsi en historiographie depuis Hérodote - on pourra analyser ou psychanalyser sa défiance vis-à-vis de l'anglophone, rejeter ses jugements sur Papineau, Bourassa, Paul Gouin, Mgr Charbonneau de Montréal, mais on ne pourra jamais reprocher à Groulx de ne pas avoir dit clairement ses opinions. Un homme debout ! Un prêtre aussi, profondément fidèle à l'essentiel de sa vie. Il a pourtant appris à Valleyfield et ailleurs tout ce qu'il y a d'indiscret et de mal-éduqué chez certains confrères quand le pouvoir et la vanité s'en mêlent. Vigoureuse et tenace comme lui, sa croyance est sans illusion, sans faux espoir, mais il connaît trop son Eglise pour en douter. Est-ce le temps de la quitter parce que la mer est houleuse ! C'est qu'il aime la tempête: parce qu'elle signifie pour lui la vie, la vraie vie. Aurait-il comme par hasard, ainsi qu'on l'a souvent dit, subordonné sa foi à son nationalisme, son sacerdoce à sa vocation d'entraîneur de la jeunesse ! Les Mémoires montrent qu'il s'est souvent posé la question. Ne devrait-on pas dire plutôt que la foi chez lui tient lieu de phare, et à l'occasion de rétroviseur ? Par contre il nous a paru plus nettement nationaliste à la manière québecoise dans ses Mémoires que dans ses livres, quoique sa manière «québe- 
coise », issue de l'humanisme des années 1900, ne se réduit jamais à une solution "unique", politique, linguistique, religieuse ou économique. Il veut tout à la fois, sans isoler, sans mềme créer de priorités. Ce que Groulx souhaite avant tout le reste, il me semble du moins, c'est la création en Amérique d'un Etat français autonome dont Québec serait pour le moment l'instrument plutôt que le but. La Confédération de 1867 serait une étape à franchir le plus tôt possible. Pour ce faire Québec doit prendre les devants au nom même de cet Etat français : "Peu à peu, malgré tout, les Canadiens français apprendront à se tourner vers le Québec comme vers le pôle naturel de leur vie politique et nationale »... ... Point de survivance d'une minorité française au Canada qui soit pensable sans le Québec...

Les Mémoires indiquent que Lionel Groulx fut malgré les apparences un homme mesuré qui aura creusé sa route à mesure en vérifiant au jour le jour le pour et le contre de ses options immédiates, sans jamais, cependant, broncher autour d'une triple option fondamentale, que nous énumérerons selon l'ordre alphabétique pour ne désorienter personne: une foi, une langue, un pays.

Certaines de ses pages décevront sûrement. Leurs défauts sautent aux yeux autant que leurs inséparables qualités : souci trop conscient du personnage historique qu'il fut, des jugements précipités, des hypothèses de travail, qui appellent des dossiers plus critiques que des propos de fumoirs et des conversations de sacristie. Groulx ne se défie pas assez de ses amis; il lit trop unilatéralement Le Devoir comme une Bible quotidienne. Il oublie de tenir compte des autres media d'information. Il moralise, il idéalise, enjolive comme aujourd'hui on sociologise et on politise. Ses appels à la Providence risquent parfois d'être un peu fatalistes. Il y a aussi des répétitions, des faux recoupages comme quand un texte de 1930 vient expliquer une attitude de 1920; certaines de ses citations sont trop longues pour être d'une littérature de Mémoires; son plaisir à pardonner à ceux dont il triomphe est très évident. Il en veut trop aux politiciens pour ne pas avoir voulu en être un, et meilleur que les autres. Quand il trace le portrait d'un Bourassa "idéal", on en est tout prêt de dire que ce qui l'a déçu chez l'autre est ce qu'il aurait voulu être lui-même s'il avait opté pour une autre voie que la sienne.

Retenons qu'il s'agit bel et bien de Mémoires. Le genre a ses lois, Groulx les connaît : un cadre biographique, le respect de la chronologie, des portraits, des tableaux d'ensemble, des digressions, priorité du témoin oculaire et de la tradition orale sur la 
source écrite. Mais pouvait-il faire autrement, dans les circonstances, que de servir ses souvenirs les plus percutants à travers l'histoire de ses livres et le haut-parleur d'une renommée bien méritée ?

Maintenant le pire qui pourrait arriver à ses pages finales, et le pire arrivera sûrement, c'est de tomber sous des yeux agités, pressés, en quête d'une hypothèse à confirmer, d'une idée à vendre, d'une impatience à justifier. Je pense aux interviewers toujours pressés d'aller ailleurs. Qui osera lire à la suite au-delà de 1600 pages imprimées ? C'est plus court d'en parler. Parce qu'il a beaucoup écrit et aussi longtemps qu'il n'y aura pas de spécialistes de sa pensée, nous continuerons à le lire par extraits, hors de contexte. Et tout sera perdu, je pense surtout aux Mémoires: le ton intérieur, la tendresse, la gentille désinvolture d'un homme passionné, cette droiture inaliénable devant sa propre vie, sa fidélité essentielle aux grandes options de sa jeunesse.

Quoi qu'il arrive, la publication des Mémoires du chanoine Groulx trois ans à peine après sa mort, constitue un événement majeur de l'historiographie canadienne et peut-être aussi de l'historiographie française. Quel honneur pour un peuple encore jeune de se donner un tel homme, un écrivain aussi parfait et aussi bien identifié avec les siens, fin, cultivé, ardent et fier ! Quel humaniste en plus ! Tout ce fonds greco-latin qui est partout et qui l'appuie, qui d'entre nous maintenant eût pu le mettre en œuvre aussi bien que lui ? Lionel Groulx reste le géant de l'histoire de nos lettres.

Pour la Nème fois souhaitons-nous tous ensemble, par une souscription publique s'il le faut, - allons ! messieurs du Devoir, vous lui devez bien cela - une édition nationale des Oeuvres complètes de Lionel Groulx: une grande et belle édition critique, infolio, fidèle au texte original, chargée de notes et de variantes qui disent la vie des mots et rappellent celle de l'idéologue qu'il fut. Toutes les grandes nations, toutes les grandes religions savent se payer ce luxe. Pourquoi pas nous ? Tant de jeunes historiens pourraient se mettre au travail tout de suite. Dans ce cas, les Mémoires constitueraient le premier tome idéal de ces opera omnia, puisqu'ils nous offrent à l'avance, grâce au manuscrit fidèlement typographié par Fides, une vue panoramique authentique signée par nul autre que Lionel Groulx lui-même. 